\title{
«NUEVOS DERECHOS»Y PRINCIPIO DE AUTODETERMINACIÓN ENTRE EL TRIBUNAL EUROPEO DE DERECHOS HUMANOS, LA CORTE CONSTITUCIONAL ITALIANA Y EL LEGISLADOR NACIONAL. ALGUNAS REFLEXIONES
}

\author{
Maria Grazia Rodomonte
}

\begin{abstract}
SUMARIO: 1. El ARTÍCULO 8 DEL CONVENIO EUROPEO DE DERECHOS HUMANOS Y EL FORTALECIMIENTO DE LAS POSICIONES JURÍDICAS SUBJETIVAS JURÍDICAMENTE TUTELADAS 2. LOS «NUEVOS» DERECHOS COMO DERECHOS DE ORIGEN JURISPRUDENCIAL Y LA TUTELA MULTINIVEL DE LOS DERECHOS 3. AUTODETERMINACIÓN Y LECTURA «EVOLUTIVA» DE NUESTRO TEXTO CONSTITUCIONAL. LAS DECISIONES DE «VALOR» COMO MÁRGENES DE LA INTERPRETACIÓN EVOLUTIVA DE LA CONSTITUCIÓN ITALIANA 4. EL ESPACIO DEL LEGISLADOR NACIONAL ENTRE MARGEN DE EVALUACIÓN, CONSENSO MAYORITARIO DE LOS ESTADOS MIEMBROS Y NUEVAS CONQUISTAS DE LA CIENCIA 5. A MODO DE CONCLUSIÓN. ALGUNAS REFLEXIONES SOBRE EL DERECHO JURISPRUDENCIAL Y EL DERECHO POSITIVO.
\end{abstract}

\section{EL ARTÍCULO 8 DEL CONVENIO EUROPEO DE DERECHOS HUMANOS Y EL FORTALECIMIENTO DE LAS POSICIONES JURÍDICAS SUBJETIVAS JURÍDICAMENTE TUTELADAS}

Como es sabido, el ámbito de aplicación del artículo 8 del Convenio Europeo de Derechos Humanos (de ahora en adelante CEDH), que establece el derecho al respeto a la vida privada y familiar ${ }^{1}$, se ha ampliado de manera

${ }^{1}$ El artículo 8 del Convenio europeo de derechos humanos tutela, como se sabe, cuatro aspectos distintos de la autonomía personal: la vida privada, la vida familiar, el domicilio y la correspondencia. Art. 8 del Convenio: «derecho al respeto de la vida privada y familiar: 1. Toda persona tiene derecho al respeto de su vida privada y familiar, de su domicilio y de su correspondencia. 2. No podrá haber injerencia de la autoridad pública en el ejercicio de este derecho sino en tanto en cuanto esta injerencia esté prevista por la ley y constituya una 
paralela a los cambios de las costumbres y de la conciencia social, al crecimiento de las necesidades individuales y a las posibilidades ofrecidas por la evolución de la ciencia médica y tecnológica. Después de un tiempo, se ha asistido a una relectura, por parte del Tribunal Europeo de Derechos Humanos (de ahora en adelante TEDH), del dato normativo contenido en el art. 8 del Convenio -referencia de hecho bastante indeterminada- que ha llevado a la creación de nuevos «insaciables» derechos ${ }^{2}$.

El progresivo reconocimiento de nuevos derechos, a nivel europeo y nacional, tiene su origen en una específica matriz cultural, es decir el derecho a la vida privada que, como ha destacado Marta Cartabia ${ }^{3}$, ha llegado a Europa desde los Estados Unidos ${ }^{4}$, se ha desarrollado rápidamente hacia el derecho a la autonomía personal o a la autodeterminación y ha generado a su vez toda una serie de nuevos derechos individuales. En este sentido hay quien, como Luca Antonini, habla de «normativización del deseo» ${ }^{5}$, expresión que, aunque sintética, sin embargo da en el blanco del nudo problemático de dicha evolución.

medida que, en una sociedad democrática, sea necesaria para la seguridad nacional, la seguridad pública, el bienestar económico del país, la defensa del orden y la prevención de las infracciones penales, la protección de la salud o de la moral, o la protección de los derechos y las libertades de los demás prevista por la ley y constituya una medida que, en una sociedad democrática, sea necesaria para la seguridad nacional, la seguridad pública, el bienestar económico del país, la defensa del orden y la prevención de las infracciones penales, la protección de la salud o de la moral, o la protección de los derechos y las libertades de los demás»».

${ }^{2}$ La expresión es de SeBoK, A.J., The insatiable Constitution, in Southern California Law Review, 70, 1996-97, 417-471, también utilizada por ANTONINI, L., Il traffico dei diritti insaziabili, Rubettino, 2008.

${ }^{3}$ De hecho es evidente que por medio de la autodeterminación se va afirmando el individualismo, es decir «una concepción individual-libertaria», como bien ha destacado MARTA CARTABIA, que es fruto de la exportación de un «producto» americano a Europa precisamente a través de una lectura «abierta» del principio de autodeterminación por parte del TEDH. La autora recuerda de hecho que «si tratta delle stesse matrici culturali e giuridiche che sono alla base di tutta la stagione dei diritti individuali di matrice liberal che ha segnato una intera epoca del costituzionalismo americano: l'origine -almeno dal punto di vista giuridico- risale agli Stati Uniti degli anni ' 60 , una società e un ordinamento giuridico che in quegli anni attraversavano le storiche stagioni delle battaglie per i diritti civili. E' la rivoluzione dei costumi, soprattutto dei costumi sessuali, che ha portato sul piano giuridico alla comparsa del diritto alla privacy, intesa non come mera non interferenza nelle cose private, ma come libertà positiva o diritto all'autodeterminazione individuale». Véase M. CARTABIA, Nuovi diritti, en statoechiese.it, 2011.

${ }^{4}$ Nania, R., La libertà individuale nella esperienza costituzionale italiana, Torino, 1989, 157, marca la distancia de la Corte constitucional italiana en la sentencia. n. 28 de 1975 desde esta matriz cultural, en particular desde el derecho a la autodeterminación de la mujer en materia de aborto en los términos delineados por la Corte suprema estadounidense.

5 Antonini, L., Autodeterminazione nel sistema dei diritti costituzionali, en D'Agostino, F., Autodeterminazione. Un diritto di spessore costituzionale?, Atti del Convegno nazionale dell'UGCI, Pavia, 5-7 de diciembre 2009, Milano, 2012, 12. 
Para comprender el fenómeno en cuestión -es decir en qué medida el artículo 8 del Convenio, por medio de la jurisprudencia del TEDH, se ha convertido en el fundamento para el reconocimiento y la tutela de las posiciones individuales- es suficiente recordar lo ocurrido con la tutela de las posiciones subjetivas en el ámbito de la procreación, con particular referencia a la Procreación Médicamente Asistida (PMA).

En este contexto, se ha destacado cómo el derecho al pleno desarrollo de la personalidad ha funcionado como "catalizador normativo»" de las nuevas instancias subjetivas (o de pareja) originadas por el desarrollo social y tecnológico, con la consecuencia de que hoy se asiste en este contexto a un proceso de progresiva, y aparentemente imparable, ampliación del ámbito de aplicación del artículo 8 del Convenio. De hecho, es evidente que «una volta riconosciuto il diritto a procreare, la riconduzione all'ambito applicativo dell'articolo 8 CEDU delle sue articolazioni funzionali sembra essere- logicamente prima che giuridicamente -inevitabile» ${ }^{7}$. Del reconocimiento del derecho a la procreación, a partir de la sentencia del TEDH Evans c. United Kingdom de 2007, deriva el derecho a ser padres genéticos, según lo establecido en la sentencia Dickson; a partir del derecho a ser padres genéticos deriva a su vez el derecho a acceder a las técnicas de PMA (S.H. c. Austria); este último enlaza con el derecho (deseo) de procrear un hijo que no padezca enfermedades genéticas de las que los padres sean portadores (como se ha establecido en la sentencia Costa y Pavan), es decir el derecho a tener un hijo sano.

En fin, una auténtica concatenación de nuevos derechos que se desarrolla por medio de las decisiones del TEDH y que es posible por el hecho de que, según la interpretación del mismo Tribunal, el derecho a la privacy contiene implícitamente también el derecho a tener un hijo.

\section{LOS «NUEVOS» DERECHOS COMO DERECHOS DE ORIGEN JURISPRUDENCIAL Y LA TUTELA MULTINIVEL DE LOS DERECHOS}

El art. 8, frecuentemente en relación con el art. 14 del mismo Convenio, o sea el principio de no discriminación, suele ser interpretado como una cláusula abierta por medio de la cual pueden originarse un número potencialmente ilimitado de nuevos derechos. En este sentido, dos son los aspectos que merecen ser destacados en relación al proceso de creación de nuevos derechos.

${ }^{6}$ Penasa, S., La Corte Europea dei Diritti dell'Uomo di fronte al fattore scientifico: analisi della recente giurisprudenza in materia di procreazione medicalmente assistita e interruzione volontaria di gravidanza, en forumquadernocostituzionali.it, 3 de abril 2013.

7 ID., $I b$. 
En primer lugar se trata de derechos no escritos, es decir no explicitados en una Carta o en una Declaración, siendo derechos de origen jurisprudencial, fruto de la interpretación llevada a cabo por el TEDH a partir del art. 8 del CEDH. Como derechos no escritos no tienen por lo tanto limitaciones, exceptuando las que surgen de una eventual ponderación con otros derechos por parte del mismo Tribunal de Estrasburgo; se trata, en otros términos, de derechos absolutos 8 .

En segundo lugar, como es notorio, el proceso de imparable y progresivo reconocimiento de nuevos derechos a nivel europeo - es decir a nivel supranacional- está destinado a reflejarse a nivel nacional, precisamente por el hecho de que existe ya un complejo sistema de tutela multinivel de derechos por el cual lo que ocurre a nivel europeo impacta también a nivel interno. Sin detenernos más en el tema, es evidente hoy que cualquier decisión sobre derechos individuales tomada en sede europea tiene repercusiones en el ordenamiento nacional ${ }^{9}$. Es suficiente recordar aquí cómo, con las sentencias «gemelas» nn. 348 y 349 de 2007, la Corte constitucional italiana ha expresado su competencia para utilizar el CEDH como parámetro -en cuanto norma interpuesta en virtud del art. 117, apartado 1, de la Constitución- en el juicio de constitucionalidad de las leyes italianas y cómo a nivel interpretativo ha sido favorecido el «diálogo» entre jueces ordinarios, Corte constitucional y TEDH. Aún se podría decir más sobre el tema ${ }^{10}$, destacando que son precisamente los jueces comunes, que en este ámbito han obtenido un diálogo privilegiado con el TEDH, los más sensibles a una relectura del catálogo constitucional de los derechos por medio de la «lente» de la autodeterminación. De todas maneras, es suficiente aquí subrayar cómo el complejo mecanismo de tutela de los derechos que ve involucrados a los jueces ordinarios nacionales, a los Tribunales constitucionales, a la Corte de justicia de la Unión europea y al Tribunal europeo de derechos humanos, está llevando al centro del sistema a este último, «punto di riferimento sempre più autorevole sia delle istituzioni dell'Unione Europea sia delle istituzioni nazionali» ${ }^{11}$.

${ }^{8}$ CARTABIA, M., op. cit., 13-14.

9 ID., Ib., 3.

10 Sobre el tema véase Randazzo, B., Giustizia costituzionale sovranazionale. La Corte europea dei diritti dell'uomo, Milano, 2012.

${ }^{11}$ CARTABIA, M., op. cit., 8. Es evidente que dicha circularidad del diálogo tiene un doble efecto: por un lado representa una clara señal de la erosión de la soberanía estatal en cuanto es indudable que la tutela de los derechos se ha trasladado desde el nivel nacional al nivel europeo; además, a nivel interno pone en una posición de primer plano a los jueces comunes que pueden puentear a la Corte constitucional en cuanto sujetos directamente dialogantes con los Tribunales europeos. 


\section{AUTODETERMINACIÓN Y LECTURA «EVOLUTIVA» DE NUESTRO TEXTO CONSTITUCIONAL. LAS DECISIONES DE «VALOR» COMO MÁRGENES DE LA INTERPRETACIÓN EVOLUTIVA DE LA CONSTITUCIÓN ITALIANA}

El proceso que acabamos de describir, gracias también a la circularidad del diálogo entre los jueces, los Tribunales nacionales y los Tribunales supranacionales, originado por la tutela multinivel de los derechos, favorece la emersión de una idea libertarian del ser humano que, como se ha dicho, nace en Estados Unidos y se difunde en Europa a través de las decisiones del TEDH y que, no obstante, parece estar distante de la idea del ser humano presente en las Constituciones europeas surgidas tras el segundo conflicto mundial. Por cierto, en esta perspectiva no se quiere negar que los derechos contenidos en nuestro texto constitucional, a diferencia de la legislación ordinaria, se presten a una lectura evolutiva capaz de garantizar una duración en el tiempo del dato constitucional ${ }^{12}$. Sin embargo, esta constatación no parece por sí misma ser suficiente para afirmar que nuestro texto constitucional se preste a una lectura ilimitadamente abierta. De hecho, aquellos mismos valores que son traducidos en principios en nuestro texto constitucional representan igualmente límites y/o factores de orientación respeto a una ampliación ilimitada del número de los derechos mismos. Entre estos ciertamente cabe destacar el principio de igualdad y dignidad humana, el principio personalista y el principio pluralista ${ }^{13}$. En definitiva, parece que se pueda afirmar que la Constitución italiana no ha optado por una «insostenible neutralidad», sino que ha llevado a cabo una elección precisa en favor de algunos valores. Elección en la que es posible identificar una matriz liberal de la libertad a la que se acompañan una matriz católica y una socialista.

Por tanto, la idea de libertad entendida como «no impedimento» de la autodeterminación del individuo ${ }^{14}$ está presente en las mismas raíces del texto constitucional, aunque está destinada a convivir con otros valores condicionantes. Lo que implica que el dato constitucional no está destinado a ser una realidad inmutable, ajena al contexto social en el que opera, sino que representa un elemento «vivo», cuya vocación evolutiva queda sin embargo

12 Véase Gemma, G., Non diritti «insaziabili», ma micro-diritti costituzionali circa l'uso del proprio corpo, en Dir. e Soc., 2/2013, 213.

13 AnTonini, L., Autodeterminazione nel sistema dei diritti costituzionali, cit., considera que «sembra quindi pacifico che il ricorso al principio di autodeterminazione, se isolato dai singoli diritti, non aiuta affatto una ricostruzione coerente, attuale e rispettosa del dato costituzionale. Estremamente influenzato dalla logica individualista estranea alla Costituzione italiana, esso racchiude una forza espansiva che non tiene conto di alcuni dati salienti del nostro contesto costituzionale, tra i quali il personalismo, il principio democratico e, se si vuole, quello dell'uguale dignità».

14 Gemma, G., op. cit., 220. 
siempre y de todas formas firmemente basada en los valores en los que se funda el núcleo inmutable de nuestra Constitución.

\section{EL ESPACIO DEL LEGISLADOR NACIONAL ENTRE MARGEN DE EVALUACIÓN, CONSENSO MAYORITARIO DE LOS ESTADOS MIEMBROS Y NUEVAS CONQUISTAS DE LA CIENCIA}

En un contexto constitucional caracterizado por la posible ampliación de los derechos, no se puede no coincidir con la afirmación según la cual «oggi appare quanto mai urgente porre un freno al 'diluvio' di diritti fondamentali, riconosciuti persino in capo alle persone giuridiche, che piovono dal cielo di una Convenzione «strumento vivente» a loro tutela, Convenzione tesa ad intercettarne evoluzioni e mutazioni nel contesto giuridico, economico, storico e culturale europeo» ${ }^{15}$.

Es necesario, por lo tanto, preguntarse sobre cómo enfrentar la progresiva erosión de valores que aquel «diluvio» puede implicar; valores que si bien están presentes en la Carta constitucional, corren el riesgo de ser arrastrados por la desbordante afirmación jurisprudencial de nuevos «insaciables» derechos.

Una solución podría o - mejor dicho- debería consistir en la intervención del legislador, que podría constituir como un dique de contención efectivo frente a la tendencia antes destacada. Toda otra solución que vea -como sin embargo ocurre a menudo a nivel europeo- la preponderancia del derecho jurisprudencial sobre el derecho positivo es motivo de incertidumbre puesto que no hay duda de que «ciascun giudice uomo-solo, a seconda della sua precomprensione del caso, del suo modello valoriale di riferimento, della sua interpretazione della Costituzione, può offrire soluzioni diverse, quando non opposte, ai medesimi casi $\rangle^{16}$. Por lo tanto, cuando se tenga que decidir en ámbitos «éticamente sensibles», debería prevalecer la elección del legislador orientada a mediar entre diferentes exigencias, sopesando entre los distintos valores que entran en juego con respecto a «casos difíciles», como son ciertamente los que afectan a la procreación asistida, a la eutanasia o a las adopciones homoparentales. En este sentido, se puede recordar lo afirmado por

15 Randazzo, B., La bulimia della Corte dei «desideri», en forumquadernicostituzionali, 14 de mayo de 2013, quien además destaca (ID., Ib., 3): «Si osservi, en passant, che la moltiplicazione dei diritti individuali produce come effetto non secondario la corrispondente espansione del potere della Corte, che accresce la propria competenza (ex art. 32 CEDU) in modo sostanzialmente incontrollabile e può finire per essere arbitrario».

${ }^{16}$ Así TrIPODINA, C., Nascere e morire tra diritto politico e diritto giurisprudenziale, en Cavino, M. - Tripodina, C., La tutela dei diritti fondamentali tra diritto politico e diritto giurisprudenziale: «casi difficili» alla prova, Milano, 80. 
los tres jueces del TEDH que han expresado su opinión disidente en la reciente decisión Gross c. Suiza con respecto a la petición de suicidio asistido. En esta ocasión los jueces han recordado que «Where, however, there is no consensus between the States Parties to the Convention, either as to the relative importance of the interest at stake or as to the best means of protecting it, particularly where the case raises sensitive moral or ethical issues, the margin will be wider. By reason of their direct and continuous contact with the vital forces of their countries, the State authorities are, in principle, in a better position than an international court to give an opinion, not only on the 'exact content of the requirements of morals' in their country, but also on the necessity of a restriction intended to meet such requirements $\rangle^{17}$. Las palabras de los jueces -además de no ser aisladas ya que en otras ocasiones el TEDH tuvo la oportunidad de marcar las competencias de los Estados miembros en materia de garantía de los derechos ${ }^{18}$ - demandan el papel de la política nacional como sede prioritaria para definir los contenidos de las elecciones éticas $\mathrm{y}$, diremos nosotros, como dique efectivo frente a las indicaciones procedentes del TEDH y de la lectura «individualista» de los derechos que surge en su jurisprudencia. De modo que precisamente el reconocimiento de un «margen de apreciación ${ }^{19}$ en favor de los Estados miembros debería permitirles llevar a cabo elecciones diferenciadas en relación a los «casos difíciles» en el ámbito bioético.

También en este caso cabe hacer algunas observaciones.

En primer lugar merece la pena detenerse sobre la hipótesis de que el TEDH perciba la existencia de un consenso no unánime sino «mayoritario» entre los Estados parte del Convenio europeo. En la decisión Vallianatos y otros contra Grecia (ric. n. 29381/09, 32684/09) de 7 de noviembre 2013 en tema de reconocimiento jurídico de las parejas homosexuales ${ }^{20}$, es significativa la actitud del TEDH al evaluar el consenso social europeo hacia al reconocimiento de las parejas homosexuales. Los Jueces de Estrasburgo, de hecho, sobre todo en esta ocasión, han subrayado la existencia de un trend emergente a nivel europeo, llegando a identificar cuáles son efectivamente los Países que

17 Véase el considerando 7 de la opinión disidente conjunta de los jueces Raimondi, Jočienė y Karakaş.

18 Véase VARI, F., Considerazioni critiche a proposito della sentenza Costa et Pavan della II sezione della Corte EDU, en Rivista AIC, 1/2013, 6, quien recuerda lo que se ha evidenciado con motivo de la Declaración final del Congreso de Brighton de 2012 sobre el futuro del Tribunal de Estrasburgo, es decir, el papel del TEDH es subsidiario respecto a la garantía de los derechos a nivel nacional, así como las afirmaciones presentes repetidamente en las decisiones del mismo TEDH.

19 Sobre el margen de apreciación véase TANZELla, P., Il margine di apprezzamento, en CARTABIA, M. (ed.), I diritti in azione, Bologna, 2007, 145 ss.

${ }^{20}$ Valenti, V., Dalla Cedu una tutela 'per direttissima' delle coppie omosessuali, en Confronti costituzionali, 30 de diciembre 2013. 
tutelan jurídicamente las parejas homosexuales. Lo que ha inducido al TEDH a efectuar un balance entre derechos, a utilizar un strict scrutiny con respecto a las motivaciones aducidas por el Estado y, por lo tanto, a falta de firmes argumentaciones, a declarar la violación del Convenio. A este respecto se puede convenir con la observación según la cual lo que más impresiona de las argumentaciones aducidas por el TEDH es, sin duda, el hecho de que cuando el Tribunal presta atención a la evolución social y a las transformaciones en acto en la mayoría de los Estados europeos confirma «ancor di più il ruolo della Convenzione europea quale living instrument, il fatto cioè che si tratti di un testo normativo che richiede di essere interpretato alla luce delle evoluzioni della società europea contemporanea ${ }^{21}$. Además, en las motivaciones de los jueces de Estrasburgo emerge, aunque con mucha cautela, «l'intenzione di 'chiudere il cerchio' in merito all'art. 8 della Convenzione» ${ }^{22}$, es decir, la intención de fijar un nivel mínimo de tutela jurídica de las parejas homosexuales que permita de condicionar, a la luz de un consenso social europeo en construcción, la discrecionalidad de los legisladores nacionales. Precisamente este intento del Tribunal europeo de asegurar un nivel mínimo de garantía en favor de las parejas homosexuales ha conducido a considerar que, desde este punto de vista, el legislador nacional que tiene la intención de introducir la disciplina de un instituto por medio del cual reconocer jurídicamente las parejas «non potrà escludere, da tale riconoscimento, le coppie omosessuali $\rangle^{23}$.

Por otro lado, incluso en materia de procreación, ámbito al que se ha hecho referencia al principio de estas páginas, es evidente cómo de la jurisprudencia del TEDH emergen ulteriores limitaciones del margen de valoración acordado al legislador nacional. En particular, parece que pueda hablarse, en este contexto, de un verdadero «gravamen procedimental» puesto sobre el legislador nacional en referencia a las conquistas de la ciencia médica. De hecho, «pur trattandosi di un trend giurisprudenziale ancora in via di consolidamento, ma che trova significative sponde a livello di giurisprudenza costituzionale nazionale (Italia e Francia), sembra che la Corte EDU sia venuta ad individuare un ulteriore criterio per definire il margine di apprezzamento, che diviene, in sede di scrutinio dell'esercizio di tale margine, anche un parametro di valutazione (almeno potenziale, cfr. S.H. Grand Chamber) delle scelte discrezionali operate a livello nazionale ${ }^{24}$. El legislador nacional está de hecho llamado a probar que ha llevado a cabo, «all'interno del procedimento decisionale secondo forme variamente individuabili, una attività di raccolta, analisi e valutazione dello status quo riscontrabile a livello scienti-

21 ID., $I b$.

22 ID., $I b$.

23 Así VALENTI, V., cit.

24 PENASA, S., La Corte Europea dei diritti dell'Uomo di fronte al fattore scientifico, op. cit., 12 . 
fico-tecnologico, in modo da «introdurre» concretamente la complessità scientifica, che lo legittima verso scelte altamente discrezionali, nel processo decisionale, che ne sarà quindi condizionato, o quanto meno orientato. Il soddisfacimento di tale condizione sembra fondare una presunzione di compatibilità delle scelte discrezionali con la CEDU $»^{25}$. Es decir, el legislador resulta condicionado en la toma de decisiones políticas por el hecho de tener en debida consideración el progreso científico y tecnológico. Precisamente en la decisión S.H. contra Austria, conforme a lo sostenido por el Tribunal «spetta al legislatore prevedere dei meccanismi decisionali e normativi, $i$ quali consentano la valutazione periodica degli eventuali progressi scientifici, in modo da adeguare l'impianto normativo ai medesimi» ${ }^{26}$.

\section{A MODO DE CONCLUSIÓN. ALGUNAS REFLEXIONES SOBRE EL DERECHO JURISPRUDENCIAL Y EL DERECHO POSITIVO}

En conclusión, parece que se pueda afirmar que el espacio de intervención del legislador interno está condicionado por una evolución de la jurisprudencia del TEDH, no siempre clara y coherente por lo que respeta la evaluación del margen de apreciación relativo a la existencia de un «consenso» entre los Estados, además de la incidencia de los progresos científicos y tecnológicos sobre las decisiones del legislador. En ambos casos la efectiva discrecionalidad del legislador nacional en la ponderación de los intereses en juego parece destinada a una progresiva e inevitable limitación.

Además, no hay que olvidar cómo la circularidad del diálogo entre jueces, Tribunales constitucionales y europeos, puede terminar por condicionar las mismas posiciones asumidas por el legislador, induciéndolo, a pesar de la tendencia a no querer asumir una posición frente los asuntos éticamente sensibles, a acoger ex post posiciones ya surgidas en sede judicial, en particular a nivel europeo. A este respecto, es significativo cuanto recientemente afirmado en relación a la decisión tomada por el Tribunal de justicia de la UE en el caso Hay en tema de no discriminación por motivos de preferencia sexual, o sea que «l'azione giudiziaria, europea e nazionale, rappresenta il primo ed essenziale momento di contatto tra 'essere e dover essere', tra fenomeno sociale e realtà giuridica; il primo momento in cui si registrano quelle trasformazioni sociali, che richiedono -in questo caso, e con riguardo al contesto italiano, da tempo, troppo tempo- di diventare 'diritto legislativo'. Tuttavia, questa consapevolezza è accompagnata anche dalla convinzione che l'attivismo giudiziario, senza il successivo intervento del Legislatore, conduce alla frammenta-

${ }^{25}$ ID., $I b$.

${ }^{26}$ ID., $I b$. Aunque, más allá de las consideraciones presentes en la decisión, la Corte no ha negado de manera absoluta la compatibilidad de la decisión del legislador austriaco con el art. 8 del CEDH. 
rietà della tutela giuridica dei diritti fondamentali. Ed allora, se per provocare la risposta del Legislatore nazionale è necessario passare da Strasburgo e Lussemburgo, ben venga. Solo così, forse, sarà possibile accorciare la distanza dell'Italia dagli altri Paesi europei $\rangle^{27}$. Esta observación, más allá de que se pueda o no auspiciar el activismo judicial, evidencia cómo de todas maneras las opciones escogidas por los tribunales europeos pueden influir en los legisladores internos, sobre todo cuando estos parecen «débiles» como frecuentemente ocurre hoy en muchos países, como es el caso de Italia, frente a la crisis de «prestigio» y credibilidad originadas directamente por la crisis de representación, desde hace tiempo mal endémico de nuestro país.

Aunque indirectamente la jurisprudencia de los tribunales europeos, en particular del TEDH, condicione la legislación interna, aún mayor es el papel tradicionalmente jugado por la Corte constitucional como Corte de derechos. No puede dejarse de destacar que la así llamada tutela multinivel de los derechos determine un replanteamiento del papel jugado por la Corte constitucional cuando opera como Corte de los derechos ${ }^{28}$; lo que no dejar de tener en cuenta el impacto del derecho europeo y del derecho supranacional en la redefinición del sistema de las fuentes que a su vez es una señal tangible del repensamiento del Estado y de la soberanía nacional. Sin embargo, la Corte constitucional debe cumplir la tarea, de la cual no debería sustraerse, de ser el garante de los derechos en los espacios que le son encomendados. No se puede negar, de hecho, que incluso el juez constitucional interno, tras la «constitucionalización» de las normas CEDH conforme al sentido en el cual son interpretadas por la jurisprudencia del TEDH, dispone de un espacio no indiferente de intervención.

En realidad, el juez constitucional no ha dejado de manifestar en diferentes ocasiones una especie de regresión en su papel decisorio, como en el caso de la ordenanza n. 150 de 2012 en materia de procreación heteróloga, con la que la Corte ha preferido remitir los actos a los jueces de quibus ${ }^{29}$ antes que

27 VALENTE, V., Verso l'europeizzazione del diritto nazionale di famiglia? (Brevi osservazioni a margine del caso Hay, Corte di Giustizia UE, C-267/12, sent. 12 de diciembre 2013), en forumquadernicostituzionali, 24 de febrero 2014. Cursiva nuestra.

${ }^{28}$ Según Valente, V., Verso l'europeizzazione del diritto nazionale di famiglia? (Brevi osservazioni a margine del caso Hay, Corte di Giustizia UE, C-267/12, sent. 12 diciembre 2013), en forumquadernicostituzionali, 24 de febrero de 2014, se asiste a una verdadera europeización del derecho nacional de familia. De hecho «Come sembra stia facendo la CEDU nel dare applicazione del diritto convenzionale, così i Giudici di Lussemburgo sembrano intenzionati ad operare essi stessi un controllo più stringente sulla discrezionalità degli Stati Membri al fine di garantire, in modo più rigoroso, l'uniformità nell'applicazione e nell'interpretazione del diritto dell'UE specie in un ambito in cui si registra una forte disomogeneità tra i diversi Paesi Membri, nella definizione dei livelli di tutela delle coppie omosessuali».

${ }^{29}$ En dicha ordenanza la Corte ha subrayado el carácter preliminar del art. 117, apartado 1, de la Constitución, de hecho subordinando el examen de la cuestión de legitimidad 
entrar en el fondo del asunto. En esta ocasión no solo la Corte parece mostrar una actitud de excesiva prudencia hacia una decisión del TEDH, aun tratándose de una fuente sub-constitucional, sino que incluso parece indudable que se ha abstenido de decidir, «provocando un'alterazione del carattere incidentale del giudizio costituzionale, che invece si proponeva, apparentemente, di difendere $\rangle^{30}$. Además, con respecto a las relaciones con el Tribunal de Estrasburgo, si se considera la tendencia de la Corte constitucional a no enfrentarse a cuestiones internas, otorgando prioridad lógica y jurídica a la cuestión comunitaria, es decir, una peligrosa asimilación de las cuestiones de legitimidad constitucional en la resolución de las cuestiones comunitarias, resulta claro el riesgo que la Corte corre de perder su prestigio y, más en general, la posibilidad misma de verificar la legitimidad constitucional de las normas internas $^{31}$.

Sin embargo, llamada otra vez a pronunciarse sobre la constitucionalidad de la prohibición de fecundación heteróloga contenida en la ley n. 40 de 2004, la cual ya antes había sido objeto de censura por parte del juez constitucional y objeto de numerosas decisiones judiciales, la Corte constitucional no sólo ha censurado dicha prohibición, sino que también se ha orientado en la dirección de una clara delimitación de las posibles opciones del legislador en un ámbito en el que, según lo establecido por el juez constitucional en la reciente decisión n. 162 de 2014, no podría negarse un espacio de autodeterminación de la pareja en mérito a la decisión de formar una familia y de volverse padres.

La intervención, esta vez «a voces», de la Corte constitucional podría ser explicada precisamente por la voluntad de recuperar un espacio en aquella dinámica de la tutela multinivel que corre el riesgo de «reducir» a la Corte

constitucional a una ulterior intervención de los jueces a quibus que pueden volver a formular la cuestión de legitimidad por violación de la norma supranacional interpuesta. De hecho podemos estar de acuerdo con cuantos en la doctrina expresan perplejidades frente la decisión del juez constitucional de remitir los actos al juez a quo y de considerar la decisión del TEDH como ius superveniens, aún sin definirlo como tal. Se afirma de hecho (I. PELLIZZONE, Sentenza della Corte europea sopravvenuta e giudizio di legittimità costituzionale: perché la restituzione degli atti non convince. Considerazioni a margine dell'ord. N. 150 del 2012 della Corte costituzionale, in Rivista AIC, 3/2012, 2) que «la decisione presenta alcune forzature e pare che si presti ad essere letta come una risposta alla discutibile esigenza, avvertita nel caso concreto dai giudici costituzionali, di posticipare la delicata decisione nel merito della legittimità costituzionale del divieto di fecondazione eterologa. Non sarebbe, del resto, la prima volta che la categoria delle decisioni di restituzione degli atti per ius superveniens viene utilizzata impropriamente per necessità dilatorie».

30 Pellizzone, I., op. cit., 4.

${ }^{31}$ GARNIER, T., Rinvio pregiudiziale interpretativo e giudizio di legittimità costituzionale. Nuovi scenari e nuove prospettive nel crocevia sovranazionale, en Diritto e Società, 2/2013, 250 . 
constitucional a una posición marginal y subordinada a los Tribunales europeos, en particular al Tribunal de Estrasburgo. Por tanto, se puede suponer que, mientras la Corte constitucional tolera la intervención de los jueces internos, solicitada además por ella misma frente a la demanda de interpretación conforme también al CEDH, por otra parte parece ser menos propensa a aceptar que su margen de intervención sea limitado directamente por el $\mathrm{TEDH}^{32}$ el cual, además, no ha dejado en más de una ocasión de actuar como Tribunal al que las partes pueden acudir directamente ${ }^{33}$.

Si es esta la explicación de la intervención hecha en esta ocasión por la Corte constitucional -es decir, no querer dejar espacios de acción en favor del TEDH-, parece sin embargo que puede afirmarse que el juez constitucional ha terminado por medirse, aun sólo implícitamente, con la jurisprudencia de aquel mismo Tribunal, acogiéndola sin reservas y, de hecho, confirmando, aun con otras modalidades, la tendencia a retroceder frente la jurisprudencia del TEDH. De hecho, aun sin referirse expresamente a la jurisprudencia del TEDH sobre los art. 8 y 14 del Convenio, sino más bien basándose únicamente sobre parámetros internos, accede a una lectura de la autodeterminación cuyo principio absoluto y cuya fuente inagotable de nuevos derechos en favor de los individuos y de la pareja llega también a la idea de que el derecho a la autodeterminación contiene implícitamente el derecho a tener un hijo. Desde esta lectura resulta inevitable la consecuencia de que la ponderación entre los diferentes valores en juego consiste de hecho en un «desequilibrio» en favor de la determinación de la pareja a tener un hijo, calificada por la misma Corte como «incoercible» y por lo tanto no limitable.

Además, el carácter absoluto del principio de autodeterminación -con referencia a la familia y al derecho a ser padres como fruto de una interpretación evolutiva del favor familiae fundada en el art. 31 de la Constitución-permite considerar de primordial importancia las exigencias de tutela de la salud física y psíquica de la pareja afectada por infertilidad o esterilidad total. Según el

${ }^{32}$ Sorrenti, V.G., Gli effetti del garantismo competitivo: come il sindacato di legittimità costituzionale è tornato al suo giudice naturale (a margine di Corte cost. sent. $n$. 162/2014), www.giurcost.org, 6-7.

${ }^{33}$ Como ha ocurrido no solamente en el más notorio caso Costa - Pavan (sobre el cual véase lo afirmado por SORRENTI, G., op. cit.) sino que en otras ocasiones como en el caso Hay. Es extremamente interesante la opinión parcialmente disidente presente en dicha sentencia del juez Pinto de Albuquerque, quien ha destacado que «The Grand Chamber has inaugurated a novel remedy in the present judgment, which posits a specific legislative solution to a social problem that has allegedly not been solved by the national legislator after the persons concerned have taken direct action before the Court. The Court is no longer a mere "negative legislator»: it assumes the role of a supra-national "positive legislator» which intervenes directly in the face of a supposed legislative omission by a State Party», casi que fuese una «Corte constitucional europea» «functioning as a positive legislator at the direct request of the persons concerned». 
juez, de hecho, la imposibilidad de formar una familia con hijos puede afectar de manera negativa «in misura anche rilevante, sulla salute della coppia, nell'accezione che al relativo diritto deve essere data $»^{34}$. La discrecionalidad del legislador ordinario se ve totalmente excluída cuando se trata de elegir en relación a la oportunidad de las terapias que tengan que ser aplicadas -en este caso a parejas en las que uno de los partner sea afectado por infertilidad o esterilidad total. La discrecionalidad del legislador, ya limitada por la libertad de autodeterminación de la pareja, tiene que retroceder, según la Corte, frente al hecho de que no es el legislador sino el médico quien tiene que tomar las decisiones más oportunas en cuanto idóneas a garantizar la tutela del derecho a la salud física y psíquica de los que componen la pareja.

En fin, hay que subrayar cómo según la Corte sigue habiendo límites al derecho a tener una familia con hijos, de manera que la fecundación heteróloga puede ser legítimamente utilizada por las parejas afectadas por infertilidad o esterilidad absoluta, ya que, además, no se permite la maternidad subrogada. Sin embargo, una vez abiertas las puertas a una «reescritura» de la ley por parte de los jueces, nada impide que, en el futuro, sobre la base de la autodeterminación, se admita la posibilidad de que ulteriores límites marcados por el legislador de la ley n. 40 de 2004 sean superados, en lo relativo al derecho a la salud y a las decisiones tomadas por el juez constitucional sobre la racionalidad de la ley.

En conclusión, se puede afirmar que, a pesar de todo tipo de consideraciones en relación a la citada decisión de la Corte constitucional ${ }^{35}$, lo que más sorprende cuando se examine la jurisprudencia del TEDH y de nuestra Corte constitucional sobre los temas éticamente sensibles es la progresiva reducción del margen de elección del legislador, es decir, la cesión del derecho positivo en favor del derecho jurisprudencial. El legislador nacional, desde luego no exento de críticas sobre la inercia que demasiadas veces afecta su actuación, parece «restringido» en un circuito en el que hoy prevalece la actividad de «reescritura» del derecho por los jueces -desde los jueces comunes hasta las Cortes europeas pasando por las Cortes constitucionales- todos, aunque variadamente ${ }^{36}$, protagonistas indiscutibles de un pluralismo juris-

${ }^{34}$ Véase el considerando n. 7 de la sentencia de la Corte constitucional núm. $162 / 2014$.

35 Véanse, para comentarios de primera lectura, además de SORRENTI, G., cit.; MorroNE, A., Ubi scientia ibi iura, en www.giurcost.org, 2014; RUGGERI, A., La Consulta apre all'eterologa ma chiude, dopo averlo preannunziato, al «dialogo» con la Corte Edu, en forumquadernicostituzionali.it, 2014; sea permitida la referencia a RoDOMONTE, M.G., E' un diritto avere un figlio?, en RodOMONTE, M.G., confronti costituzionali.eu, 17 de junio 2014.

${ }^{36}$ Variadamente porque, como se ha ya observado, al «centro» del sistema de tutela multinivel parece ser siempre más colocado precisamente el TEDH con su jurisprudencia (en este sentido véase lo sostenido retro, par. 2). Tribunal que parece desempeñar una función «nomofiláctica» respeto a la definición de los contornos de los «nuevos derechos». 
prudencial que parece asumir un papel prevalente respecto a las elecciones de los órganos representativos. Sin duda la citada sentencia de la Corte constitucional prueba claramente que el papel del legislador nacional está limitado a un ámbito de acción que es difícil delinear, limitado, en el contexto de la tutela multinivel, entre el respecto a la autodeterminación, las conquistas progresivas de la ciencia y de la técnica y el juicio sobre la racionalidad de la ley.

En pocas palabras, un legislador reducido al silencio o, más bien, que corre el riesgo de hablar con una sola voz, la del derecho jurisprudencial, ya que sigue un recorrido marcado por la interpretación judicial. Un legislador al que sin embargo sería oportuno restituir, por las razones antecedentemente destacadas, espacios de intervención y capacidad de decisión además de la reputación necesaria para tomar decisiones difíciles en los «casos difíciles».

\section{Traducción por SimÓn PedRo IsAZA}

TITLE: 'New rights' and principle of self-determination between the European Court of Human Rights, the Constitutional Italian Court and the national legislator. Some reflections.

RESUMEN: El artículo 8 del CEDH establece el derecho al respeto de la vida privada y familiar, que ha sufrido una relectura expansiva a través de una enriquecedora jurisprudencia de las divergentes instancias judiciales, y sobre todo, de las resoluciones emanadas por el relevante Tribunal Europeo de Derechos Humanos, que impactan, de forma directa e indiscutible, en los planos domésticos.

Así, resulta innegable cómo el sentir de la jurisprudencia europea se impone, y sirve de impulsor de la redefinición de los sistemas, y del propio Derecho, lo cual suscita las reticencias de ciertos tribunales nacionales que se sienten limitados por la línea marcada por el TEDH. Al mismo tiempo, resultará necesario replantear el papel del legislador que, quizá, se vea demasiado inspirado, por no decir condicionado, por los razonamientos jurisprudenciales.

PALABRAS CLAVE: Derechos humanos, principio de autodeterminación, Corte Constitucional, legislador, Tribunal Europeo de Derechos Humanos.

ABSTRACT: The article 8 of the ECHR establishes the right to respect for private and family life that has suffered an expansive rereading by means of an enriching jurisprudence of the different judicial instances, and especially of the resolutions adopted by the relevant European Court of Humans Rights that impact directly and indubitably in the domestic levels. 
Thus, it is undeniable how the feel of the European jurisprudence is imposed, and uses as impeller of the redefinition of the systems, and as the own law, which provokes the reluctances of certain national courts that feel limited by the direction marked by the ECHR. At the same time, it will be necessary to restate the role of the legislator who, probably, seems to be too much inspired, for not saying determined, for the jurisprudential reasoning.

KEYWORDS: Human Rights, Principle of self-determination, Constitutional Court, legislator, European Court of Humans Rights.

RECIBIDO: 11.11 .2014 ACEPTADO: 26.11.2014 